\title{
Neural-Based Compensation of Nonlinearities in an Airplane Longitudinal Model with Dynamic-Inversion Control
}

\author{
YanBin Liu, ${ }^{1}$ YuHui Li, ${ }^{2}$ and FeiTeng Jin $^{2}$ \\ ${ }^{1}$ Key Laboratory of Unmanned Aerial Vehicle Technology of Ministry of Industry and Information Technology, Nanjing University of \\ Aeronautics and Astronautics, Nanjing 210016, China \\ ${ }^{2}$ College of Astronautics, Nanjing University of Aeronautics and Astronautics, Nanjing 210016, China
}

Correspondence should be addressed to YanBin Liu; nuaa_liuyanbin@139.com

Received 4 January 2017; Accepted 29 November 2017; Published 19 December 2017

Academic Editor: Silvia Conforto

Copyright (C) 2017 YanBin Liu et al. This is an open access article distributed under the Creative Commons Attribution License, which permits unrestricted use, distribution, and reproduction in any medium, provided the original work is properly cited.

\begin{abstract}
The inversion design approach is a very useful tool for the complex multiple-input-multiple-output nonlinear systems to implement the decoupling control goal, such as the airplane model and spacecraft model. In this work, the flight control law is proposed using the neural-based inversion design method associated with the nonlinear compensation for a general longitudinal model of the airplane. First, the nonlinear mathematic model is converted to the equivalent linear model based on the feedback linearization theory. Then, the flight control law integrated with this inversion model is developed to stabilize the nonlinear system and relieve the coupling effect. Afterwards, the inversion control combined with the neural network and nonlinear portion is presented to improve the transient performance and attenuate the uncertain effects on both external disturbances and model errors. Finally, the simulation results demonstrate the effectiveness of this controller.
\end{abstract}

\section{Introduction}

For a general longitudinal model of the airplane, the flight control law tends to be designed in terms of the linearized model corresponding to the given trim points. On this basis, the proportional-integral-derivative (PID) controller is used to achieve the desired flight performance under the assumption that the short-period dynamics are faster than the phugoid mode [1]. However, the classical PID controller may be limited due to too many parameters that need to be scheduled and optimized for the strong coupling airplane model under the complicated flight condition. As a result, the inversion design approach is a very useful tool in the control design [2], and the main advantage lies in avoiding the iterative regulation concerning the control parameters, and this controller provides greater flexibility for the strong coupling system [3]. More importantly, the control design using the dynamic-inversion method is based on the nonlinear model instead of the interpolated linear model [4].

In some studies, the inversion control design is realized by adopting feedback signals to offset inherent coupling dynamics, thus guaranteeing the satisfactory decoupling control ability. In particular, an investigation example was illustrated using the dynamic-inversion methodology for the linear model of a generic X-38 type reentry vehicle [5]. Correspondingly, the closed-loop stability and robustness of a dynamic-inversion flight controller for reentry vehicles were quantified in consideration of the influence along with the different flight dynamics. In addition, a methodology was presented using a combination of the linear dynamicinversion controller and adaptive filter in order to implement MIMO reconfigurable flight control [6]. Such control design could improve significantly the tracking performance, handling qualities, and PIO tendencies for the closed system. Besides that, Doman and Ngo [7] discussed an indirect adaptive control problem by applying a baseline dynamicinversion control structure. Furthermore, a quaternion-based attitude controller was developed based on the inversion control approach for the X-33 in the ascent flight phase. The dynamic-inversion control approaches were introduced for a spacecraft, not only an airplane, to realize the attitude control in response to the servo-constraint dynamics [8]. 
This control law consisted of particular and auxiliary parts wherein the particular part played a role in driving the spacecraft attitude variables, whereas the auxiliary potion provided the necessary internal stability with the aid of the involved null-control vector. In general, the inversion method is adopted in the control design for both the airplane and spacecraft models in recent years. It is noted that the main difference between the inversion approach and conventional method lies in that the resulting design model is achieved by the state feedback, thus keeping the exact dynamics in contrast to the approximating linearization [9].

In this paper, the flight control law is proposed using the neural-based inversion design method and nonlinear compensation for a general longitudinal model of the airplane. In particular, the dynamic-inversion control can relieve the strong coupling effects regarding the model dynamics, whereas the neural-based compensation is helpful in improving the robust performance to suppress the uncertain disturbances. There are three aspects of this problem that have to be addressed. First, the inversion design method is introduced to convert the nonlinear mathematic model to the equivalent model accurately. After that, the inversion control law is designed to stabilize the system and relieve the coupling effects. Furthermore, the compensation using the neural network and nonlinear portion is introduced to improve the transient performance and system robustness. Lastly, an airplane example is provided to verify the feasibility of the proposed controller.

\section{Longitudinal Model of an Airplane}

The longitudinal motion of the airplane involves only vertical motion parameters and aerodynamic actions, so the airplane dynamics can be described based on the velocity coordinate. While the elevator deflection $\left(\delta_{e}\right)$ and throttle setting $\left(\eta_{c}\right)$ are selected as control inputs $(U)$, the airplane model with the state variables $X=[V, \gamma, q, \alpha, h]$ is given as follows [10]:

$$
\begin{aligned}
& \dot{V}=\frac{T \cos \alpha-D}{m}-g \sin \gamma, \\
& \dot{\gamma}=\frac{L+T \sin \alpha}{m V}-\frac{g \cos \gamma}{V}, \\
& \dot{q}=\frac{M_{y}}{I_{y}}, \\
& \dot{\alpha}=q-\dot{\gamma}, \\
& \dot{h}=V \sin \gamma,
\end{aligned}
$$

where $m$ and $I_{y}$ denote the mass and moment of inertia of the airplane, respectively. Besides that, the lift $L$, the $\operatorname{drag} D$, the thrust $T$, and the pitching moment $M_{y}$ are determined by

$$
\begin{aligned}
L & =\frac{1}{2} \rho V^{2} S_{w} C_{L}, \\
D & =\frac{1}{2} \rho V^{2} S_{w} C_{D},
\end{aligned}
$$

$$
\begin{aligned}
M_{y} & =\frac{1}{2} \rho V^{2} S_{w} \bar{c} C_{M}, \\
T & =\frac{1}{2} \rho V^{2} S_{w} C_{T} .
\end{aligned}
$$

In (2), $S_{w}$ and $\bar{c}$ represent the reference area and mean aerodynamic chord, respectively. Furthermore, we assume that the lift coefficient $C_{L}$, drag coefficient $C_{D}$, pitching moment coefficient $C_{M}$, and propulsive coefficient $C_{T}$ in this work are approximately stated by

$$
\begin{aligned}
C_{L} & =f_{L}(\rho, V, \alpha), \\
C_{D} & =f_{D}(\rho, V, \alpha), \\
C_{T} & =f_{T}\left(\rho, V, \alpha, \eta_{c}\right), \\
C_{M} & =f_{M_{y}}(\rho, V, \alpha, q)+f_{\delta_{e}} \delta_{e} .
\end{aligned}
$$

Also, the gravity constant $(g)$ and air density $(\rho)$ as a function of altitude are shown by

$$
\begin{aligned}
& g=f_{g}(h), \\
& \rho=f_{\rho}(h) .
\end{aligned}
$$

Based on (1)-(3), the balance restrictions are provided by

$$
\begin{aligned}
& \frac{Q f_{T}\left(f_{\rho}\left(h_{d}\right), V_{d}, \alpha_{r}, \eta_{c r}\right) \cos \alpha_{r}-Q f_{D}\left(f_{\rho}\left(h_{d}\right), V_{d}, \alpha_{r}\right)}{m} \\
& \quad=0, \\
& \frac{Q f_{L}\left(f_{\rho}\left(h_{d}\right), V_{d}, \alpha_{r}\right)+Q f_{T}\left(f_{\rho}\left(h_{d}\right), V_{d}, \alpha_{r}, \eta_{c}\right) \sin \alpha_{r}}{m V_{r}} \\
& =\frac{f_{g}\left(h_{d}\right)}{V_{d}},
\end{aligned}
$$

$Q=\frac{1}{2} f_{\rho}\left(h_{d}\right) V_{d}^{2} S_{w}$

$f_{M_{y}}\left(f_{\rho}\left(h_{d}\right), V_{d}, \alpha_{r}\right)+f_{\delta_{e}} \delta_{e r}=0$.

For any $V_{d}$ and $h_{d}$ in (5), the trim flight parameters regarding $\alpha_{r}, \eta_{c r}$, and $\delta_{e r}$ can be solved. To this end, the inversion control system, in accordance with whether accurate feedback linearization or Taylor linear approximation is used, can be designed based on these obtained trim values.

\section{Inversion Control Laws Based on Accurate and Approximate Equivalent Model}

For the nonlinear model of the airplane in (1), its inversion model can be derived by applying, respectively, the differential geometry theory and small perturbation theory. Correspondingly, the feedback linearization method transforms the nonlinear model of the aircraft to the equivalent model which keeps completely the high-order dynamics of the original 
model. As a result, not only is the resulting inversion model based on the feedback linearization more accurate than that based on the approximate linearization, but also the control capacity is enhanced in that the uncertain effects of the approximate linearization are removed accordingly [11].

3.1. Inversion Control Law Using Feedback Linearization. First, selecting $V$ and $h$ as the system outputs, the derivative of $\dot{V}$ corresponding to $Y=[V, \gamma, \alpha, \eta, h]^{T}$ is deduced based on the feedback linearization idea and differential geometry theory [12], and it is expressed by

$$
\begin{aligned}
\ddot{V} & =\frac{\omega_{1} \dot{Y}}{m}, \\
\omega_{1}^{T} & =\left[\begin{array}{c}
\omega_{1}^{1} \\
\omega_{1}^{2} \\
\omega_{1}^{3} \\
\omega_{1}^{4} \\
\omega_{1}^{5}
\end{array}\right] \\
& =\left[\begin{array}{c}
\left.\frac{\partial T}{\partial V}\right) \cos \alpha-\left(\frac{\partial D}{\partial V}\right) \\
-m g \cos \gamma \\
\left(\frac{\partial T}{\partial \alpha}\right) \cos \alpha-T \sin \alpha-\left(\frac{\partial D}{\partial \alpha}\right) \\
\left(\frac{\partial T}{\partial h}\right) \cos \alpha-\left(\frac{\partial D}{\partial h}\right)-m\left(\frac{\partial g}{\partial h}\right) \sin \gamma
\end{array}\right]
\end{aligned}
$$

where $\eta$ is the intermediate variable that needs to be adopted, so, further differentiating $\ddot{V}$ with respect to $Y$, we have

$$
\begin{aligned}
V^{(3)} & =\frac{\left(\omega_{1} \ddot{Y}+\dot{Y}^{T} \Omega_{1} \dot{Y}\right)}{m}, \\
\Omega_{1} & =\frac{d \omega_{1}}{d Y}=\left[\frac{d \omega_{1}^{1}}{d Y}, \frac{d \omega_{1}^{2}}{d Y}, \frac{d \omega_{1}^{3}}{d Y}, \frac{d \omega_{1}^{4}}{d Y}, \frac{d \omega_{1}^{5}}{d Y}\right] .
\end{aligned}
$$

Considering the presence of $\ddot{Y}=[\ddot{V}, \ddot{\gamma}, \ddot{\alpha}, \ddot{\eta}, \ddot{h}]^{T}$ in (7) and simultaneously combining it with

$$
\begin{gathered}
\ddot{\alpha}=\dot{q}-\ddot{\gamma}=\frac{M_{y}}{I_{y}}-\ddot{\gamma}=\frac{Q f_{M_{y}}(\rho, V, \alpha)+Q f_{\delta_{e}} \delta_{e}}{I_{y}}-\ddot{\gamma} \\
=\frac{Q f_{M_{y}}(\rho, V, \alpha)}{I_{y}}-\ddot{\gamma}+\frac{Q f_{\delta_{e}}}{I_{y}} \delta_{e}=C+D \delta_{e},
\end{gathered}
$$

then we have

$$
\begin{aligned}
V^{(3)} & =\frac{\left(\omega_{1} \ddot{Y}+\dot{Y}^{T} \Omega_{1} \dot{Y}\right)}{m} \\
& =\frac{\left(\omega_{1} \ddot{Y}_{0}+\dot{Y}^{T} \Omega_{1} \dot{Y}\right)}{m}+\frac{\omega_{1}^{3} D}{m} \delta_{e}+\frac{\omega_{1}^{4} B}{m} \eta_{c} \\
& =V_{0}^{(3)}+g_{11} \delta_{e}+g_{12} \eta_{c}, \\
\ddot{Y}_{0} & =[\ddot{V}, \ddot{\gamma}, C,-A \dot{\eta}-B \eta, \ddot{h}]^{T} .
\end{aligned}
$$

Equation (9) shows that the expression of $V^{(3)}$ includes the control inputs $\delta_{e}$ and $\eta_{c}$, indicating that the nonlinear model has been partially transformed into the linear system [13]. Alternatively, higher order differential equations of $h$ can be deduced as

$$
\begin{aligned}
\ddot{h}= & \dot{V} \sin \gamma+V \dot{\gamma} \cos \gamma, \\
h^{(3)}= & \ddot{V} \sin \gamma+2 \dot{V} \dot{\gamma} \cos \gamma-V \dot{\gamma}^{2} \sin \gamma+V \ddot{\gamma} \cos \gamma, \\
h^{(4)}= & V^{(3)} \sin \gamma+3 \ddot{V} \dot{\gamma} \cos \gamma-3 \dot{V} \dot{\gamma}^{2} \sin \gamma+3 \dot{V} \ddot{\gamma} \cos \gamma \\
& -3 V \dot{\gamma} \ddot{\gamma} \sin \gamma-V \dot{\gamma}^{3} \cos \gamma+V \gamma^{(3)} \cos \gamma .
\end{aligned}
$$

In (10), the second derivative of the flight path angle $\gamma$ with regard to $Y=[V, \gamma, \alpha, \eta, h]^{T}$ is written as

$$
\begin{aligned}
\ddot{\gamma} & =\pi_{1} \dot{Y}, \\
\pi_{1}^{T} & =\left[\begin{array}{c}
\pi_{11} \\
\pi_{12} \\
\pi_{13} \\
\pi_{14} \\
\pi_{15}
\end{array}\right] \\
& =\left[\begin{array}{c}
\frac{(\partial L / \partial V)+(\partial T / \partial V) \sin \alpha}{m V \quad \frac{L+T \sin \alpha}{m V^{2}}+\frac{g \cos \gamma}{V^{2}}} \\
\frac{g \sin \gamma}{V} \\
\frac{(\partial L / \partial \alpha)+(\partial T / \partial \alpha) \sin \alpha+T \cos \alpha}{m V} \\
\frac{(\partial T / \partial \eta) \sin \alpha}{m V}
\end{array}\right] .
\end{aligned}
$$

Similarly, the differentiation of $\ddot{\gamma} \operatorname{regarding} Y=$ $[V, \gamma, \alpha, \eta, h]^{T}$ is obtained by

$$
\begin{aligned}
\gamma^{(3)} & =\pi_{1} \ddot{Y}+\dot{Y}^{T} \Omega_{1} \dot{Y} \\
& =\pi_{1} \ddot{Y}_{0}+\dot{Y}^{T} \Omega_{1} \dot{Y}+\pi_{13} D \delta_{e}+\pi_{14} B \eta_{c} \\
\Omega_{1} & =\frac{d \pi_{1}}{d Y}=\left[\frac{d \pi_{11}}{d Y}, \frac{d \pi_{12}}{d Y}, \frac{d \pi_{13}}{d Y}, \frac{d \pi_{14}}{d Y}, \frac{d \pi_{15}}{d Y}\right]^{T} .
\end{aligned}
$$


Substituting (9) and (12) into (10), we have

$$
\begin{aligned}
h^{(4)}= & \frac{\left(\omega_{1} \ddot{Y}_{0}+\dot{Y}^{T} \Omega_{1} \dot{Y}\right) \sin \gamma}{m}+3 \ddot{V} \dot{\gamma} \cos \gamma \\
& -3 \dot{V} \dot{\gamma}^{2} \sin \gamma+3 \dot{V} \ddot{\gamma} \cos \gamma-3 V \dot{\gamma} \ddot{\gamma} \sin \gamma \\
& -V \dot{\gamma}^{3} \cos \gamma+V \cos \gamma\left(\pi_{1} \ddot{Y}_{0}+\dot{Y}^{T} \Omega_{1} \dot{Y}\right) \\
& +\left(\pi_{13} D \cos \gamma+\frac{\omega_{1}^{3} D}{m} \sin \gamma\right) \delta_{e} \\
& +\left(\pi_{14} B \cos \gamma+\frac{\omega_{1}^{4} B}{m} \sin \gamma\right) \eta_{c} \\
= & h_{0}^{(4)}+g_{21} \delta_{e}+g_{22} \eta .
\end{aligned}
$$

With the integration of (9) and (13), we get

$$
\left[\begin{array}{l}
V^{(3)} \\
h^{(4)}
\end{array}\right]=\left[\begin{array}{l}
V_{0}^{(3)} \\
h_{0}^{(4)}
\end{array}\right]+\left[\begin{array}{ll}
g_{11} & g_{12} \\
g_{21} & g_{22}
\end{array}\right]\left[\begin{array}{l}
\delta_{e} \\
\eta_{c}
\end{array}\right]=F_{0}+G U .
$$

If the matrix $G$ is invertible, let

$$
\left[\begin{array}{l}
V^{(3)} \\
h^{(4)}
\end{array}\right]=\left[\begin{array}{l}
v_{1} \\
v_{2}
\end{array}\right]=v
$$

where $v$ represents the so-called pseudo-control vector [14], so the inversion model of the airplane is built by

$$
U=G^{-1}\left(v-F_{0}\right)=f^{-1}(X, v) .
$$

As long as the output of (16) is regarded as the input of the airplane model and at the same time (15) holds, the decoupling control goal can be achieved for the nonlinear airplane model. Furthermore, we define tracking errors as [14]

$$
\begin{aligned}
S_{V}= & \left(\ddot{V}-\ddot{V}_{d}\right)+2 \lambda_{V}\left(\dot{V}-\dot{V}_{d}\right)+\lambda_{V}^{2}\left(V-V_{d}\right), \\
S_{h}= & \left(h^{(3)}-h_{d}^{(3)}\right)+3 \lambda_{h}\left(\ddot{h}-\ddot{h}_{d}\right)+3 \lambda_{h}^{2}\left(\dot{h}-\dot{h}_{d}\right) \\
& +\lambda_{h}^{3}\left(h-h_{d}\right),
\end{aligned}
$$

where $V_{d}$ and $h_{d}$ represent command signals, respectively. Differentiating $S_{V}$ and $S_{h}$ and simultaneously combining them with (14), we have

$$
\begin{aligned}
{\left[\begin{array}{c}
\dot{S}_{V} \\
\dot{S}_{h}
\end{array}\right]=} & {\left[\begin{array}{l}
R_{V} \\
R_{h}
\end{array}\right]+\left[\begin{array}{ll}
g_{11} & g_{12} \\
g_{21} & g_{22}
\end{array}\right]\left[\begin{array}{l}
\delta_{e} \\
\eta_{c}
\end{array}\right] } \\
R_{V}= & 2 \lambda_{V}\left(\ddot{V}-\ddot{V}_{d}\right)+\lambda_{V}^{2}\left(\dot{V}-\dot{V}_{d}\right)+V_{0}^{(3)}-V_{d}^{(3)}, \\
R_{h}= & 3 \lambda_{h}\left(h^{(3)}-h_{d}^{(3)}\right)+3 \lambda_{h}^{2}\left(\ddot{h}-\ddot{h}_{d}\right) \\
& +\lambda_{h}^{3}\left(\dot{h}-\dot{h}_{d}\right)+h_{0}^{(4)}-h_{d}^{(4)} .
\end{aligned}
$$

Let the inversion control law be [15]

$$
\left[\begin{array}{l}
\delta_{e} \\
\eta_{c}
\end{array}\right]=\left[\begin{array}{ll}
g_{11} & g_{12} \\
g_{21} & g_{22}
\end{array}\right]^{-1}\left[\begin{array}{cc}
-R_{V} & -k_{V} S_{V} \\
-R_{h} & -k_{h} S_{h}
\end{array}\right]
$$

so we have

$$
\begin{gathered}
S_{V} \dot{S}_{V}=-k_{V} S_{V}^{2}<0, \\
S_{h} \dot{S}_{h}=-k_{h} S_{h}^{2}<0 .
\end{gathered}
$$

In (20), $S_{V}$ and $S_{h}$ will converge to zero exponentially by choosing $k_{V}$ and $k_{h}$ as properly positive constants, while making track errors $V-V_{d}$ and $h-h_{d}$ reach zero rapidly [15]. Furthermore, the measurement errors in relation to the system outputs and state variables are considered in (15) and (16), and we have

$$
U=(G+\Delta G)^{-1}\left[(v+\Delta v)-\left(F_{0}+\Delta F_{0}\right)\right],
$$

where $\Delta G, \Delta v$, and $\Delta F_{0}$ are the uncertainties caused by the sensor errors. Accordingly, tracking errors in (17) change to $S_{V}+\Delta S_{V}$ and $S_{h}+\Delta S_{h}$. In this case, if the control law in (19) is selected, the Lyapunov stability in (20) may not be satisfied. Therefore, it is necessary to apply the adaptive signals to offset the uncertain effect in relation to the sensor noise as a result of ensuring the global stability throughout the overall flight envelope.

3.2. Inversion Control Law Using Approximate Linearization. The approximate linearization approach is considered that the airplane movement is associated with small deviations from the steady flight state. And all high-order dynamics are regarded to be small such that their actions are negligible in contrast to the first-order model dynamics. When the firstorder terms are kept in (1) and (5) using the approximate linearization method [16], then the following linear equations are obtained:

$$
\begin{aligned}
& \Delta \dot{X}=\left[\begin{array}{c}
\Delta \dot{V} \\
\Delta \dot{\gamma} \\
\Delta \dot{q} \\
\Delta \dot{\alpha} \\
\Delta \dot{h}
\end{array}\right] \\
& =\left[\begin{array}{ccccc}
-X_{V} & -g & 0 & -X_{\alpha} & 0 \\
Z_{V} & 0 & 0 & Z_{\alpha} & 0 \\
-M_{V} & 0 & M_{q} & -M_{\alpha} & 0 \\
-Z_{V} & 0 & 1 & -Z_{\alpha} & 0 \\
0 & V_{r} & 0 & 0 & 0
\end{array}\right]\left[\begin{array}{c}
\Delta V \\
\Delta \gamma \\
\Delta q \\
\Delta \alpha \\
\Delta h
\end{array}\right] \\
& +\left[\begin{array}{cc}
0 & X_{\eta c} \\
-Z_{\delta e} & 0 \\
M_{\delta e} & M_{\eta c} \\
Z_{\delta e} & 0 \\
0 & 0
\end{array}\right]\left[\begin{array}{c}
\Delta \delta_{e} \\
\Delta \eta_{c}
\end{array}\right]=A_{\Delta} \Delta X+B_{\Delta} \Delta U .
\end{aligned}
$$

Correspondingly, the inversion control law based on this approximate model is expressed by

$$
\left[\begin{array}{l}
\delta_{e} \\
\eta_{c}
\end{array}\right]=\left[\begin{array}{l}
\delta_{e r} \\
\eta_{c r}
\end{array}\right]+\operatorname{pinv}\left(B_{\Delta}\right)\left(\Delta v-A_{\Delta} \Delta X\right),
$$


where pinv represents the pseudo-inverse function, and let

$$
\Delta v=\left[\begin{array}{c}
k_{V p}\left(V-V_{c}\right)+k_{V d} \dot{V} \\
k_{\gamma} \theta \\
k_{q} q \\
k_{\alpha} \theta \\
k_{h p}\left(h-h_{c}\right)+k_{h d} \dot{h}
\end{array}\right]=\Delta \dot{X} .
$$

In (24), if control parameters are selected suitably, $\Delta v$ will approach $\Delta \dot{X}$ such that the inversion control based on the approximate linearization principle can be realized in the given flight condition.

\section{Robust Adaptive Control with Neural-Based Compensation of Nonlinearities}

Improving the transient performance is very important for the aircraft model to follow the expected command rapidly without deviating from the design point. Alternatively, the system robustness will guarantee flight stability with the existence of the large model uncertainties and external disturbances. As a result, the transient performance and system robustness can be an issue for the aircraft model to realize the challenging tasks.

To this end, this work combines the above dynamicinversion control with the compensation of the neural network and nonlinear potion in order to ensure system robustness and self-adaption and to improve the transient performance. This is because the inversion control is sensitive to modeling errors due to the need of the detailed knowledge of the nonlinear airplane model. In this case, the application of the neural network can alleviate this sensitivity, and the nonlinear portion can ameliorate the transient performance associated with the inversion controller [17].

First, the inversion design idea based on the feedback linearization principle transforms the nonlinear model in (1) to a standard form in (14). Correspondingly, the inverse model with the uncertain parts is expressed by

$$
U=\widehat{G}^{-1}\left(\widehat{v}-\widehat{F}_{0}\right)=\widehat{f}^{-1}(X, \widehat{v}) .
$$

Afterwards, the inversion error is defined by

$$
\varepsilon=f(X, U)-\widehat{f}(X, U) .
$$

Based on (25) and (26), (15) is rewritten as

$$
\left[\begin{array}{l}
V^{(3)} \\
h^{(4)}
\end{array}\right]=\left[\begin{array}{l}
\widehat{v}_{V} \\
\widehat{v}_{h}
\end{array}\right]=\left[\begin{array}{c}
\nu_{V}+\varepsilon_{V} \\
v_{h}+\varepsilon_{h}
\end{array}\right]=v+\varepsilon .
$$

Furthermore, the pseudo-control vector consisting of the proportional controller, command derivative, and adaptive signal is selected [18], and it is expressed as

$$
\begin{aligned}
& v_{V}=v_{V p}+V_{d}^{(3)}-\widehat{v}_{a d V}, \\
& v_{h}=v_{h p}+h_{d}^{(4)}-\widehat{v}_{a d h},
\end{aligned}
$$

where

$$
\begin{aligned}
v_{V p}= & k_{V p}\left(V_{d}-V\right)+k_{V d 1}\left(\dot{V}_{d}-\dot{V}\right) \\
& +k_{V d 2}\left(\ddot{V}_{d}-\ddot{V}\right) \\
v_{h p}= & k_{h p}\left(h_{d}-h\right)+k_{h d 1}\left(\dot{h}_{d}-\dot{h}\right)+k_{h d 2}\left(\ddot{h}_{d}-\ddot{h}\right) \\
& +k_{h d 3}\left(h_{d}^{(3)}-h^{(3)}\right) .
\end{aligned}
$$

After substituting (28) and (29) into (27), we have

$$
\begin{aligned}
e_{V}^{(3)}= & -k_{V p} e_{V}-k_{V d 1} \dot{e}_{V}-k_{V d 2} \ddot{e}_{V}+\left(\widehat{v}_{a d V}-\varepsilon_{V}\right), \\
e_{h}^{(4)}= & -k_{h p} e_{h}-k_{h d 1} \dot{e}_{h}-k_{h d 2} \ddot{e}_{h}-k_{h d 3} e_{h}^{(3)} \\
& +\left(\widehat{v}_{a d h}-\varepsilon_{h}\right) .
\end{aligned}
$$

By selecting the suitable control parameters, (30) can become Hurwitz such that the zeros of the resulting polynomial are all in the left half of the complex plane [19]. Not only that, but also the feasible selection of $\widehat{v}_{a d V}$ and $\widehat{v}_{a d h}$ can ensure that the low damping ratio is provided to achieve fast rising and regulating time when the tracking error is large. In turn, the higher damping ratio is given to decrease the overshoot when the output reaches the anticipated target. More importantly, $\widehat{v}_{a d V}$ and $\widehat{v}_{a d h}$ can further cancel the effects of uncertain errors as a result of the fact that $e_{V}$ and $e_{h}$ can approach zero and the control goal corresponding to the adaptive command track can be realized [20].

To this end, the adaptive compensation includes the nonlinear portion and output of the neural network, and it is provided as

$$
\begin{aligned}
& \widehat{v}_{a d V}=-\rho_{V} E_{V}^{T} P_{V} B_{V}+\widehat{w}_{p V}^{T} \xi_{p V}, \\
& \widehat{v}_{a d h}=-\rho_{h} E_{h}^{T} P_{h} B_{h}+\widehat{w}_{p h}^{T} \xi_{p h}, \\
& \xi_{p V}=\xi_{p h}=\exp \left(-\pi \sigma^{2}\|X-\omega\|^{2}\right),
\end{aligned}
$$

where $E_{V}=\left[e_{V}, \dot{e}_{V}, \ddot{e}_{V}\right]^{T}, E_{h}=\left[e_{h}, \dot{e}_{h}, \ddot{e}_{h}, e_{h}^{(3)}\right]^{T}, B_{V}=$ $[0,0,1]^{T}$, and $B_{h}=[0,0,0,1]^{T} \cdot \rho_{V}$ and $\rho_{h}$ denote, respectively, the designed nonpositive functions to improve the transient performance; $\xi_{p V}$ and $\xi_{p h}$ are the basis functions of the network; $\omega$ indicates its node parameter; $\widehat{w}_{p V}$ and $\widehat{w}_{p h}$ are the weights of the network; and $P_{V}$ and $P_{h}$ represent the positive definite solutions to the following Lyapunov equations:

$$
\begin{gathered}
Q_{V}=-\left(A_{V}^{T} P_{V}+P_{V} A_{V}\right), \\
Q_{h}=-\left(A_{h}^{T} P_{h}+P_{h} A_{h}\right),
\end{gathered}
$$


where $Q_{V}$ and $Q_{h}$ are selected as unit matrices, whereas

$$
\begin{aligned}
A_{V} & =\left[\begin{array}{ccc}
0 & 1 & 0 \\
0 & 0 & 1 \\
-k_{V p} & -k_{V d 1} & -k_{V d 2}
\end{array}\right], \\
A_{h} & =\left[\begin{array}{cccc}
0 & 1 & 0 & 0 \\
0 & 0 & 1 & 0 \\
0 & 0 & 0 & 1 \\
-k_{h p} & -k_{h d 1} & -k_{h d 2} & -k_{h d 3}
\end{array}\right] .
\end{aligned}
$$

In addition, the update laws of the weights $\widehat{w}_{p V}$ and $\widehat{w}_{p h}$ are adopted as [21]

$$
\begin{aligned}
& \dot{\hat{w}}_{p V}=-\gamma_{V} E_{V}^{T} P_{V} B_{V} \xi_{p V}, \\
& \dot{\widehat{w}}_{p h}=-\gamma_{h} E_{h}^{T} P_{h} B_{h} \xi_{p h},
\end{aligned}
$$

where $\gamma_{V}$ and $\gamma_{h}$ represent the positive numbers, respectively. By applying the neural network outputs to compensate uncertain errors, the steady tracking performance will be ameliorated and the system robustness will be enhanced accordingly [22].

Remark 1. Let $\widehat{v}^{*}$ be the best approximation with respect to $\varepsilon$ where $\rho=\left[\rho_{V}, \rho_{h}\right], E=\left[E_{V}, E_{h}\right], P=\left[P_{V}, P_{h}\right], B=$ $\left[B_{V}, B_{h}\right]$, and the error bound is defined as

$$
\left\|\varepsilon-\widehat{v}^{*}\right\| \leq \tau \text {. }
$$

Also, the errors between $\widehat{v}=\widehat{w}_{p}^{T} \xi_{p}$ and $\widehat{v}^{*}$ are provided as

$$
\begin{aligned}
\widehat{v}-\widehat{v}^{*} & =\widetilde{w}_{p}^{T} \xi_{p}, \\
\widetilde{w}_{p} & =\widehat{w}_{p}-\widehat{w}_{p}^{*},
\end{aligned}
$$

where $\widehat{w}_{p}^{*}$ is the weight with regard to $\widehat{v}^{*}, \widehat{v}=\left[\widehat{v}_{p V}, \widehat{v}_{p h}\right]^{T}$, $\widehat{w}_{p}=\left[\widehat{w}_{p V}, \widehat{w}_{p h}\right]^{T}$, and $\xi_{p}=\left[\xi_{p V}, \xi_{p h}\right]^{T}$. After substituting (31) and (36) into (30), we have

$$
\dot{E}=A E+B \rho E^{T} P B+B \widetilde{w}_{p}^{T} \xi_{p}+B\left(\widehat{v}^{*}-\varepsilon\right),
$$

where $A=\left[A_{V}, A_{h}\right], P=\left[P_{V}, P_{h}\right]$. Furthermore, the Lyapunov function is defined as

$$
L=\frac{1}{2} E^{T} P E+\frac{\widetilde{w}_{p V}^{T} \widetilde{w}_{p V}}{2 \gamma_{V}}+\frac{\widetilde{w}_{p h}^{T} \widetilde{w}_{p h}}{2 \gamma_{h}} .
$$
[18]

After taking the derivative with respect to (39), we obtain

$$
\begin{aligned}
\dot{L} & =-\frac{1}{2} E^{T} Q E+E^{T} \rho P B B^{T} P E+E^{T} P B\left(\widehat{v}_{a d}^{*}-\varepsilon\right) \\
& \leq-\frac{1}{2}\|E\|^{2}+\bar{\lambda}\left(\rho P B B^{T} P\right)\|E\|^{2}+\tau\left|E^{T} P B\right| \\
& \leq-\frac{1}{2}\|E\|^{2}+\tau\left|E^{T} P B\right| \\
& \leq-\frac{E^{T} P E}{2 \bar{\lambda}(P)}+\tau \sqrt{E^{T} P E} \sqrt{\bar{\lambda}(P)} .
\end{aligned}
$$

Equation (40) is negative with

$$
\sqrt{E^{T} P E}>2 \tau[\bar{\lambda}(P)]^{3 / 2} .
$$

Therefore, when $\tau \rightarrow 0$, then $\lim _{t \rightarrow 0} E \rightarrow 0$. On this basis, the nonlinear model becomes inaccurate when the airplane deviates from the design point, as a result of the fact that the inversion controller may be ineffective due to the unknown model information. In this case, the compensation output based on the neural network can cancel the model uncertainty and disturbance effect depending on the online adjustment of the weights, and the nonlinear portion can improve the transient performance, thus ameliorating global stability and self-adaptability for the overall system.

Remark 2. These functions, $\rho_{V}$ and $\rho_{h}$, change from 0 to the large negative numbers as the tracking error approaches zero [23]. At the initial condition, when controlled outputs $e_{V}$ and $e_{h}$ are far from the step commands, $\rho_{V}$ and $\rho_{h}$ are small because the influence of these nonlinear portions is constrained. In turn, when the track errors $e_{V}$ and $e_{h}$ reach the anticipated commands, in this case the nonlinear portion will become effective. In other words, $\rho_{V}$ and $\rho_{h}$ can guarantee large damping ratios of the closed system as controlled outputs reach the desired commands. To this end, the overshoot of the output response concerning the aircraft model will be reduced accordingly.

In general, the flight control law using $\rho_{V}$ and $\rho_{h}$ can achieve fast rising time for large tracking errors first. Once the system output approaches the step command, high damping ratio is set to remove the overshoot [24]. This achieves the following: not only can the flight velocity and altitude asymptotically track the step reference, but also the resulting closed-loop system can achieve better tracking performances and stronger robustness than those with the control law designed without the neural network and nonlinear part.

In particular, the structure diagram of this robust adaptive control system is shown in Figure 1.

Figure 1 shows us that the designed control system includes the inversion control with Hurwitz, the nonlinear portion, and the adaptive compensation of neural networks. Among them, the inversion control is used to relieve the coupling dynamics of the nonlinear model, the nonlinear portion improves the transient performance, and the neural network output is applied to improve system robustness and adaptability. In brief, this proposed control law can not only provide the satisfactory control performance, but also ensure the system robustness over the overall flight envelope.

\section{Illustrative Example}

In this study, the airplane properties are used in [22]. The required aerodynamic coefficients and propulsive parameters are adopted based on [13], as well. Also, the relations between 


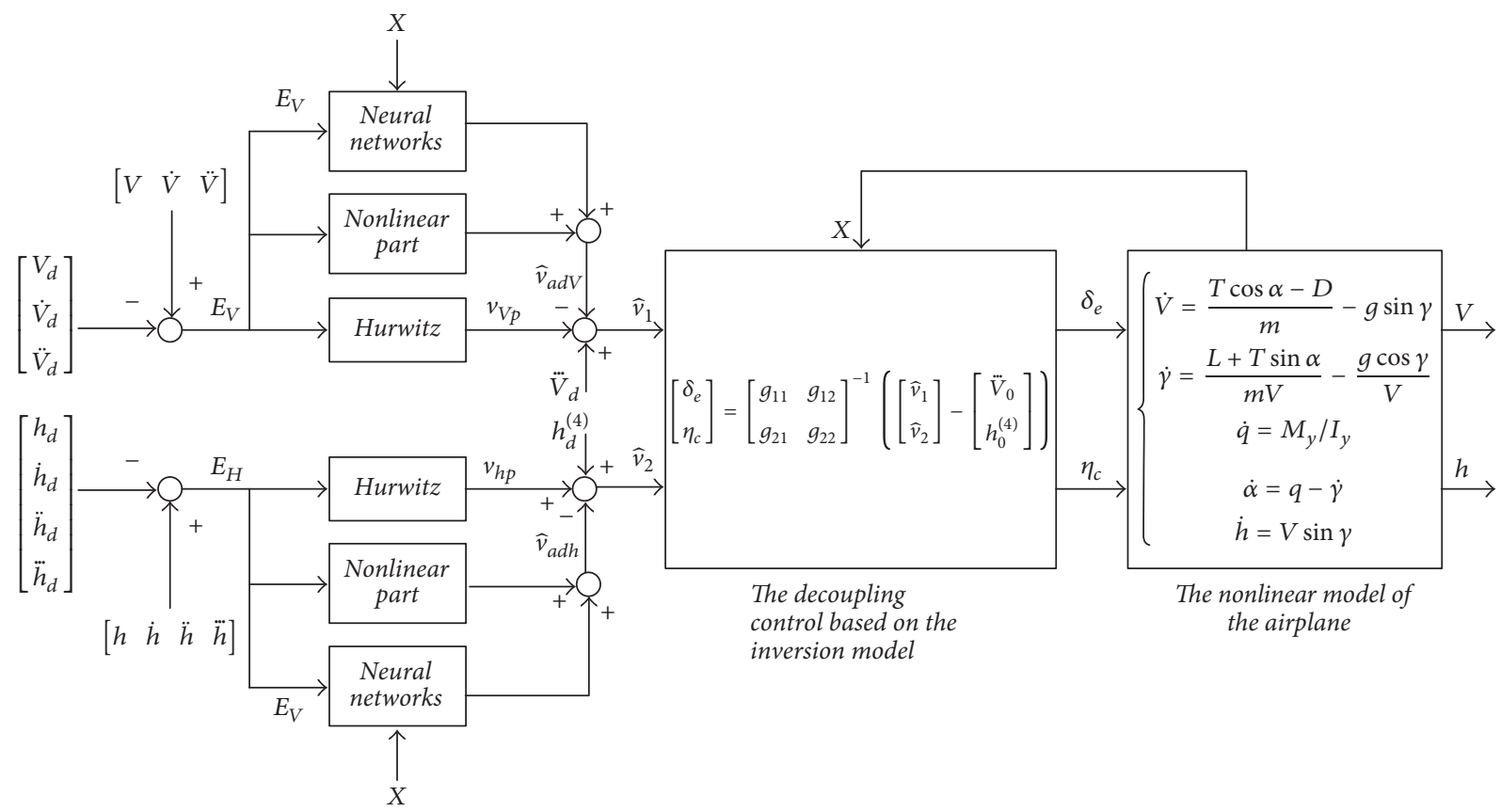

FIGURE 1: Structure diagram of robust adaptive control for the airplane.

the acceleration of gravity and air density corresponding to the altitude are approximately provided by

$$
\begin{aligned}
& \rho=\rho_{0} e^{-h / h_{0},} \\
& g=g_{0}\left(\frac{R_{0}}{R_{0}+h}\right)^{2},
\end{aligned}
$$

where $\rho_{0}=1.2266 \mathrm{~kg} / \mathrm{m}^{3}, h_{0}=73152, g_{0}=9.8 \mathrm{~m} / \mathrm{s}^{2}$, and $R_{0}=6356766 \mathrm{~m}$. Furthermore, we select the flight range as $V \in[4500 \mathrm{~m} / \mathrm{s}, 4700 \mathrm{~m} / \mathrm{s}]$ and $h \in[33500 \mathrm{~m}, 34000 \mathrm{~m}]$. According to (5), the resulting trim states can be obtained. These states alter with the change in the different altitude and velocity in order to satisfy the balance condition. In addition, any small perturbation will lead to the divergence of the flight states in relation to the unstable and nonminimum phase dynamics [25], and designing a suitable control law is critical to ensure system stability and to relieve the coupling effects of the nonlinear model dynamics [26].

First, the control goal is that the speed $V$ and altitude $h$ can follow rapidly the desired step commands $\Delta V_{c}=40 \mathrm{~m} / \mathrm{s}$ and $\Delta h_{c}=50 \mathrm{~m}$ from the trimmed condition, respectively. During the response process at the first 200 seconds, the track results using the proposed controller are displayed in Figure 2.

Figure 2 shows that the track response without the neural network and nonlinear portion is undesirable, including the large track error, slow response time, and unintended overshoot, whereas the track qualities with only the neural network compensation can be improved, but the transient performance is not good. In comparison, when using the controller with the neural network and nonlinear portion, the velocity and altitude converge to the desired commands after 30 seconds, and this indicates that the proposed control law guarantees the decoupling and tracking performances of the closed system, and simultaneously the transient performance is satisfactory, including small steady-state error, rapid response time, and less overshoot.

Furthermore, the change curves corresponding to the angle of attack and control inputs are demonstrated in Figure 3.

From Figure 3, the angle of attack changes from the initial trim value to the anticipated value rapidly, and the elevator deflection and throttle setting vary smoothly and reasonably when the controller with the neural network and nonlinear portion is applied. These results illustrate the effectiveness of the presented controller to realize the decoupling control goal. Also, the compensation outputs of the neural network are demonstrated in Figure 4.

Figure 4 shows us that the compensation outputs of the neural network change adaptively with the system output, thus improving the accuracy concerning the inverse control. Particularly, as long as the track response reaches the new trim state, the resulting compensation signals will converge to the steady values such that flight stability can be guaranteed accordingly.

Furthermore, we assume that the model parameter matrix $G$ in (14) is uncertain. In particular, the model parameters may change $20 \%$ or more due to the engineairframe coupling action, mass loss, and sensor noise. To this end, we further consider the $20 \%$ random uncertainties of model parameters, and the proposed controller can solve the tracking control problem and tolerate the larger plant uncertainty. Thus, it is expressed by

$$
g_{11}^{*}=g_{11}\left(1+\Delta_{11}\right)=\frac{\omega_{1}^{3} Q f_{\delta_{e}}}{m I_{y}}\left(1+\Delta_{11}\right)
$$




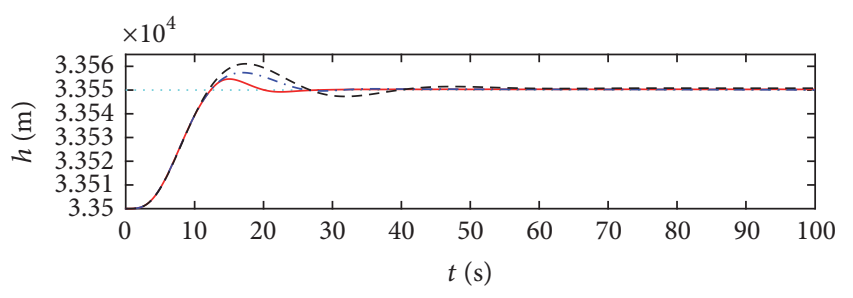

The altitude command

_ The altitude track using neural network and nonlinear portion

- - - The altitude track using neural network

- - - The altitude track without neural network and nonlinear portion

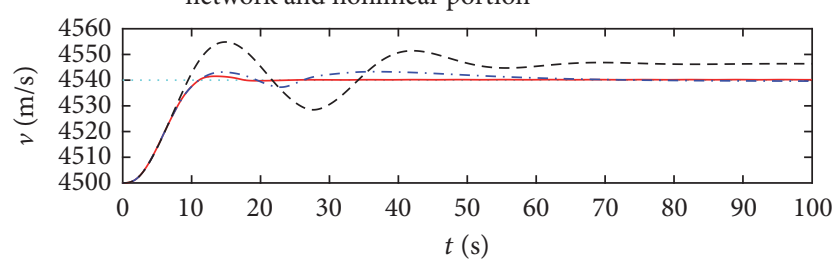

The velocity command

_ The velocity track using neural network and nonlinear portion

- - - The velocity track using neural network

- - - The velocity track without neural network and nonlinear portion

FIGURE 2: Response curves with regard to command signals.

$$
\begin{aligned}
g_{12}^{*} & =g_{12}\left(1+\Delta_{12}\right)=\frac{\omega_{1}^{4} B}{m}\left(1+\Delta_{12}\right), \\
g_{21}^{*} & =g_{21}\left(1+\Delta_{21}\right) \\
& =\left(\pi_{13} D \cos \gamma+\frac{\omega_{1}^{3} D}{m} \sin \gamma\right)\left(1+\Delta_{21}\right), \\
g_{22}^{*} & =g_{21}\left(1+\Delta_{22}\right) \\
& =\left(\pi_{14} B \cos \gamma+\frac{\omega_{1}^{4} B}{m} \sin \gamma\right)\left(1+\Delta_{22}\right),
\end{aligned}
$$

where $\Delta_{11}, \Delta_{12}, \Delta_{21}, \Delta_{22}$ are the uncertain items resulting from the sensor noise, modeling errors, inaccurate aerodynamic parameters, and so on. At this time, the presented control law with the neural network and nonlinear portion is adopted, and the simulation results accordingly can be exhibited in Figures 5 and 6.

Figures 5 and 6 show that the altitude and velocity follow the command signals quickly even in the large uncertain condition. Such results explain that the proposed controller can suppress the uncertain disturbances and guarantee system stability.

\section{Conclusion}

This paper proposes a control law using the neural-based inversion design approach with the nonlinear compensation for a general longitudinal model of the airplane. First, the
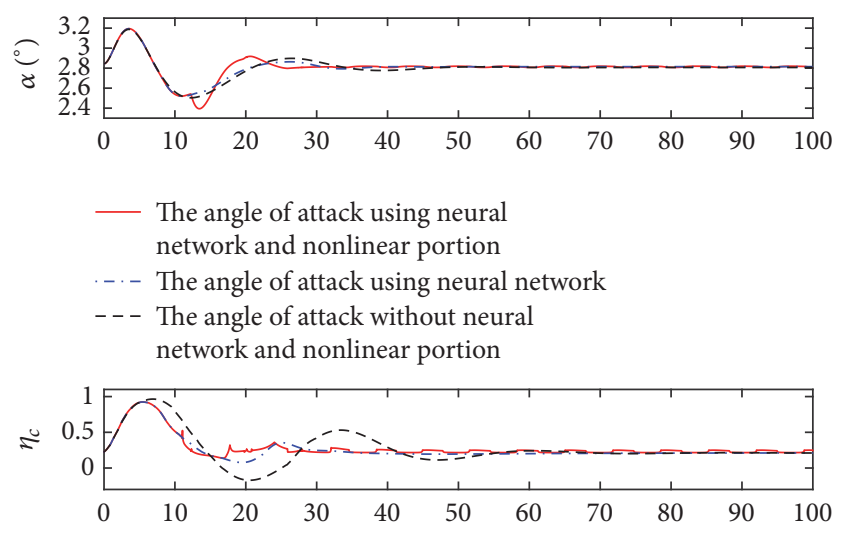

_ The propulsive input using neural network and nonlinear portion

...- The propulsive input using neural network

- - - The propulsive input without neural network and nonlinear portion

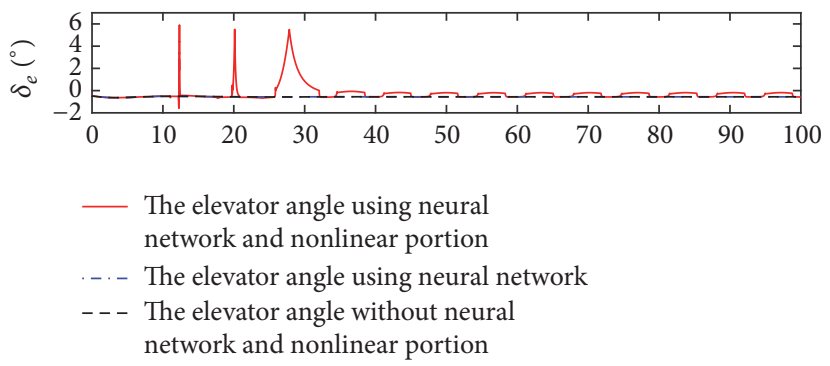

FIGURE 3: Change curves of angle of attack and control inputs.


FIGURE 4: Compensation outputs.

nonlinear model of the airplane is established, and the balance equation is gotten for the given altitude and velocity. Next, the inversion control law is designed based on the feedback linearization principle. Furthermore, the control law in combination with the neural network and nonlinear portion is proposed. For this controller, the inversion control can realize the decoupling operation concerning the nonlinear model dynamics, whereas the adaptive outputs of the 

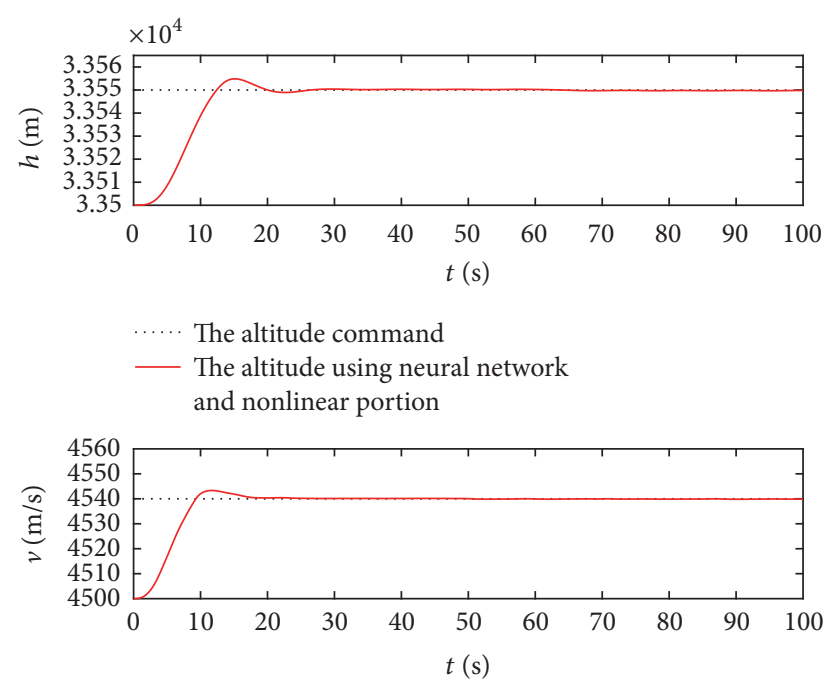

The velocity command

— The velocity track using neural network and nonlinear portion

FIGURE 5: Response curves using adaptive control law in the uncertain condition.
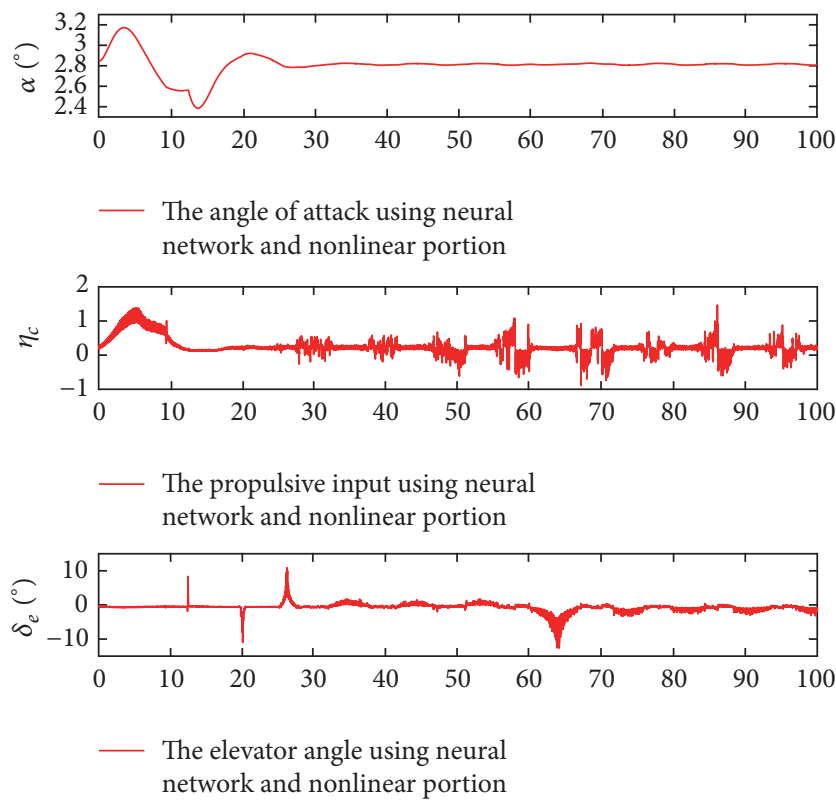

FIGURE 6: Change curves of angle of attack and control inputs in the uncertain condition.

neural network and nonlinear portion can improve system robustness, transient performance, and adaptability. Finally, the simulation is conducted to show that the proposed control methods are feasible for a general longitudinal model of the airplane.

\section{Conflicts of Interest}

The authors declare that there are no conflicts of interest regarding the publication of this paper.

\section{Acknowledgments}

This work is supported by the Fundamental Research Funds for the Central Universities (no. NJ20160052).

\section{References}

[1] Y. Liu and Y. Lu, "Collaborative deformation design using control integrated analysis methods for hypersonic waverider," International Journal of Aerospace Engineering, vol. 2015, Article ID 510414, 9 pages, 2015.

[2] B. Yang, N. Chen, L.-B. Cao, K.-M. Tse, and H.-P. Lee, "1331. Experiment and simulation studies on sound insulation performance of the wooden component," Journal of Vibroengineering, vol. 16, no. 5, pp. 2319-2329, 2014.

[3] W. Siwakosit, S. A. Snell, and R. A. Hess, "Robust flight control design with handling qualities constraints using scheduled linear dynamic inversion and loop-shaping," IEEE Transactions on Control Systems Technology, vol. 8, no. 3, pp. 483-494, 2000.

[4] S. Wang, Y. Zhang, Y. Jin, and Y. Zhang, "Neural control of hypersonic flight dynamics with actuator fault and constraint," Science China Information Sciences, vol. 58, no. 7, 2015.

[5] B. Xu, C. Yang, and Y. Pan, "Global neural dynamic surface tracking control of strict-feedback systems with application to hypersonic flight vehicle," IEEE Transactions on Neural Networks and Learning Systems, vol. 26, no. 10, pp. 2563-2575, 2015.

[6] X. Cheng, G. Tang, P. Wang, and L. Liu, "Predictive sliding mode control for attitude tracking of hypersonic vehicles using fuzzy disturbance observer," Mathematical Problems in Engineering, vol. 2015, Article ID 727162, 13 pages, 2015.

[7] D. B. Doman and A. D. Ngo, "Dynamic inversion-based adaptive/reconfigurable control of the X-33 on ascent," Journal of Guidance, Control, and Dynamics, vol. 25, no. 2, pp. 275-284, 2002.

[8] A. H. Bajodah, "Generalised dynamic inversion spacecraft control design methodologies," IET Control Theory \& Applications, vol. 3, no. 2, pp. 239-251, 2009.

[9] R. R. Da Costa, Q. P. Chu, and J. A. Mulder, "Reentry flight controller design using nonlinear dynamic inversion," Journal of Spacecraft and Rockets, vol. 40, no. 1, pp. 64-71, 2003.

[10] R. Esmaelzadeh, A. Naghash, and M. Mortazavi, "Near optimal re-entry guidance law using inverse problem approach," Inverse Problems in Science and Engineering, vol. 16, no. 2, pp. 187-198, 2008.

[11] N. Wang, X.-M. Yao, and W.-S. Li, "Nonlinear disturbanceobserver-based sliding mode control for flexible air-breathing hypersonic vehicles," Mathematical Problems in Engineering, vol. 2015, Article ID 675659, 15 pages, 2015.

[12] C. I. Marrison and R. F. Stengel, "Design of robust control systems for a hypersonic aircraft," Journal of Guidance, Control, and Dynamics, vol. 21, no. 1, pp. 58-63, 1998.

[13] B. Tian, R. Su, and W. Fan, "Multiple-time scale smooth second order sliding mode controller design for flexible hypersonic vehicles," Proceedings of the Institution of Mechanical Engineers, Part G: Journal of Aerospace Engineering, vol. 229, no. 5, pp. 781791, 2015.

[14] H. Xu, M. D. Mirmirani, and P. A. Ioannou, "Adaptive sliding mode control design for a hypersonic flight vehicle," Journal of Guidance, Control, and Dynamics, vol. 27, no. 5, pp. 829-838, 2004. 
[15] H. Sun, Z. Yang, and B. Meng, "Tracking control of a class of non-linear systems with applications to cruise control of airbreathing hypersonic vehicles," International Journal of Control, vol. 88, no. 5, pp. 885-896, 2015.

[16] R. Colgren, S. Keshmiri, and M. Mirmirani, "Nonlinear tendegree-of-freedom dynamics model of a generic hypersonic vehicle," Journal of Aircraft, vol. 46, no. 3, pp. 800-813, 2009.

[17] R. T. Rysdyk and A. J. Calise, "Adaptive model inversion flight control for tilt-rotor aircraft," Journal of Guidance, Control, and Dynamics, vol. 22, no. 3, pp. 402-407, 1999.

[18] A. J. Calise, S. Lee, and M. Sharma, "Direct adaptive reconfigurable control of a Tailless fighter aircraft," in Proceedings of the Guidance, Navigation, and Control Conference and Exhibit, pp. 88-97, Boston, Mass, USA, August 1998.

[19] H. Duan and S. Li, "Artificial bee colony-based direct collocation for reentry trajectory optimization of hypersonic vehicle," IEEE Transactions on Aerospace and Electronic Systems, vol. 51, no. 1, pp. 615-626, 2015.

[20] A. J. Calise, S. Lee, and M. Sharma, "Development of a reconfigurable flight control law for the X-36 tailless fighter aircraft," in Proceedings of the AIAA Guidance, Navigation, and Control Conference and Exhibit, Denver, Colo, USA, August 2000.

[21] M. Chen and B. Jiang, "Robust attitude control of near space vehicles with time-varying disturbances," International Journal of Control, Automation, and Systems, vol. 11, no. 1, pp. 182-187, 2013.

[22] E. Jiaqiang, C. Qian, H. Liu, and G.-L. Liu, "Design of the Ho robust control for the piezoelectric actuator based on chaos optimization algorithm," Aerospace Science and Technology, vol. 47, pp. 238-246, 2015.

[23] K. Peng, B. M. Chen, G. Cheng, and T. H. Lee, "Modeling and compensation of nonlinearities and friction in a micro hard disk drive servo system with nonlinear feedback control," IEEE Transactions on Control Systems Technology, vol. 13, no. 5, pp. 708-721, 2005.

[24] W. Lan, B. M. Chen, and Y. He, "On improvement of transient performance in tracking control for a class of nonlinear systems with input saturation," Systems \& Control Letters, vol. 55, no. 2, pp. 132-138, 2006.

[25] B. Xu and Z. Shi, "Universal kriging control of hypersonic aircraft model using predictor model without back-stepping," IET Control Theory \& Applications, vol. 7, no. 4, pp. 573-583, 2013.

[26] B. Yang, L. B. Cao, Y. Tang, N. Sun, K. Tse, and H. Lee, "Modal and dynamic responses of the human head-neck complex for impact applications," Journal of Vibroengineering, vol. 18, no. 7, pp. 4743-4755, 2016. 



\section{Hindawi}

Submit your manuscripts at

https://www.hindawi.com
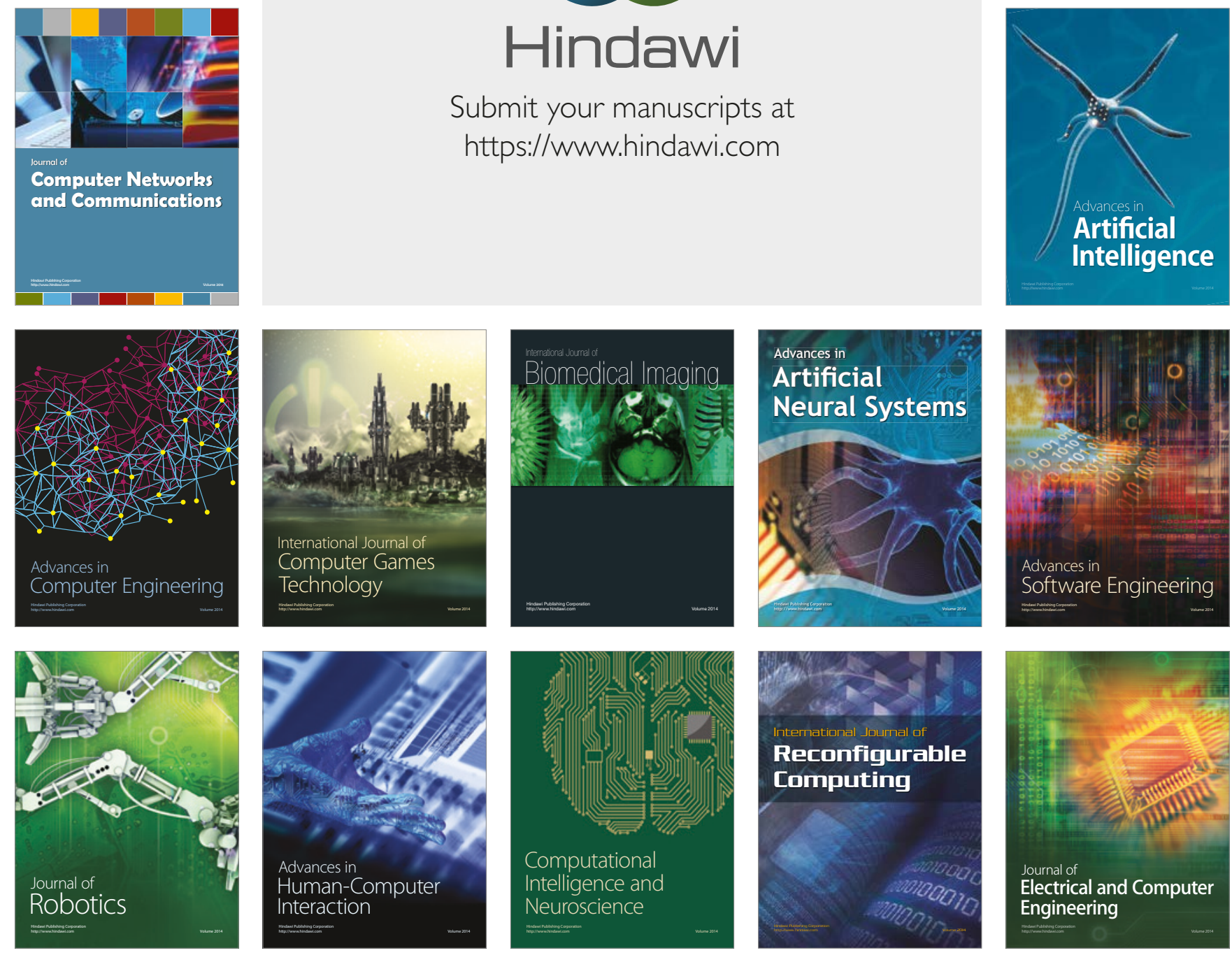